\title{
DISEÑO DE SECUENCIAS DIDÁCTICAS BLENDED LEARNING PARA EL DESARROLLO DE ESTRATEGIAS DE APRENDIZAJE Y HABILIDADES DIGITALES EN LA REINSERCIÓN A LA UNIVERSIDAD
}

\author{
Mario Martín BRIS ${ }^{1}$ \\ María del Carmen Veleros VALVERDE ${ }^{2}$ \\ Mónica García HERNÁNDEZ ${ }^{3}$
}

RESUMEN: El presente documento describe una secuencia didáctica para desarrollar habilidades de aprendizaje y de uso de tecnología en estudiantes adultos que regresan a la universidad. La propuesta integra recursos digitales en línea tales como e-books, relatos, juegos de memoria y ejercitación, en tanto las clases presenciales integran el aprendizaje colaborativo y la participación activa para asegurar la integración de este tipo de estudiante al medio universitario. La secuencia se pondrá en marcha durante este año escolar con al menos 2000 estudiantes de nuevo ingreso, se espera que logren insertarse de manera más eficiente al medio universitario después de un tiempo de distancia con su proceso educativo.

PALABRAS-CLAVE: Aprendizaje mixto. Aprendizaje situado. Educación superior para adultos.

\section{Introducción}

La integración al mundo universitario en la vida adulta, conlleva el reto de desarrollar habilidades para ser competente/competitivo en los tiempos de la tecnología digital aplicada y recuperar los hábitos de estudio previamente adquiridos.

Ser estudiante adulto es sobre todo un ejercicio de recuperación de la experiencia de vida e integración reflexiva de los saberes previos y los nuevos a la práctica profesional. Implica también generar competencias para ser autogestivo y autónomo en el trabajo escolar y asumir con ello la responsabilidad que implica el aprender. Remontar el tiempo que se ha dedicado a otras actividades en detrimento del estudio y asumir el rol de estudiante activo forman también parte de este reto.

En la primera parte del documento se presenta el planteamiento del problema, enseguida se explican los fundamentos del aprendizaje adulto, a continuación se describe el modelo propuesto se finaliza con las conclusiones.

\footnotetext{
1 Doctor en Ciencias de la Educación. UAH - Universidad de Alcalá. Madrid- España. 28801 mario.martin@uah.es

2 Doctora en Pedagogía. UNAM - Universidad Nacional Autónoma de México. UP - Universidad Panamericana. México. 03920 - cvelerosv@gmail.com

3 Doctora en Pedagogía. UNAM - Universidad Nacional Autónoma de México. UP - Universidad Panamericana. México. 03920 - monigarher@gmail.com
} 


\section{Planteamiento del problema}

Reincorporarse a la universidad después de haberse distanciado de ellos por algún tiempo debido a causas diversas como el trabajo, necesidades familiares, situaciones económicas, experiencias personales de vida entre otras, implica no solo el esfuerzo por aprender de forma eficiente sino también, remontar los hábitos de estudio y el desarrollo de otras habilidades entre las cuales puede mencionarse el trabajo en equipo, la organización del tiempo, la autorregulación, el pensamiento crítico y las habilidades digitales.

Estas carencias en el perfil de ingreso pueden eventualmente llevar a los estudiantes a abandonar nuevamente sus estudios en los primeros ciclos universitarios dificultando así la obtención del título universitario y la posible mejora en su calidad de vida derivada de la misma.

En el caso de México, la oferta de programas académicos enfocados a adultos que trabajan ha crecido exponencialmente los últimos años, ya que el segmento poblacional comprendido entre los 25 y 49 años de edad se ha incrementado en más de 4 millones del 2005 al 2010 (INEGI, 2013). Al menos 32 universidades ofrecen programas en modalidad mixta, sobre todo universidades particulares cuyos planes se componen de 33 a 60 asignaturas y se cursan de 7 a 9 ciclos cuatrimestrales. En las universidades públicas se ofrecen en su mayoría programas a distancia, cuyos planes de estudios se componen de 40 a 68 asignaturas y se cursan en 8 a 10 semestres.

Si bien no existen datos precisos y válidos respecto a la deserción de adultos trabajadores que se reincorporan a la universidad, los datos sobre deserción universitaria publicados por la Asociación Nacional de Universidades e Instituciones de Educación Superior (ANUIES, 2015), indican que en México de cada 100 alumnos que ingresan a la licenciatura, 60 terminan las materias del plan de estudios cinco años después y, de éstos, solamente 20 se reciben. Derivado de lo anterior, es factible pensar que la cifra de titulados disminuye en función de la complejidad de la vida adulta y las responsabilidades que esta conlleva.

De acuerdo con datos de la Universidad del Valle de México (2011) los estudiantes de licenciatura en este segmento, se inscriben a ella más que como una prioridad, por un anhelo de contar con el título universitario porque aspiran a una mejor posición económica y laboral. Estos estudiantes carecen de tiempo y cuentan con recursos económicos limitados, además de demandar programas que les satisfagan en términos de calidad, aprendizaje, costo y disfrute personal de la experiencia. 
A partir de la complejidad del perfil de ingreso de este tipo de estudiantes y la importancia de abatir la deserción por causas de carácter académico, se propone la configuración de secuencias didácticas enfocadas al desarrollo de habilidades digitales, así como a la recuperación y mejora de las estrategias de aprendizaje de adultos trabajadores en modalidad blended learning.

\section{Conceptos clave}

\section{El aprendizaje de los adultos}

El aprendizaje se produce como una consecuencia de la "experiencia del alumno y la interacción con el mundo". Si bien esta interacción tiene un carácter social al realizar con y para el otro, es necesario también entenderla como un proceso individual (REISER; DEMPSEY, 2007). El tipo de aprendizaje que se propone es un aprendizaje constructivo que haga posible el desarrollo personal: un aprendizaje significativo, con lógica y relacionado con los componentes cognitivos, que incida en los conceptos que el estudiante utiliza en su vida cotidiana y laboral paralela a la académica o escolar. Este aprendizaje permite descubrir, despertar e incrementar las posibilidades creativas y permite el desarrollo integral de la persona durante su vida. Entender el aprendizaje desde la significatividad del que aprende implica un cambio relativamente permanente derivado de la experiencia, que puede observarse por el uso en la práctica de nuevos conocimientos, habilidades y modificación de actitudes.

Por lo que se refiere a los fines de la educación de personas adultas se pueden mencionar de acuerdo con Knowles (1980): (1) adquirir una comprensión madura de sí misma; (2) desarrollar actitudes de aceptación y respeto hacia otras personas; (3) propiciar una actitud proactiva y adaptable al cambio; (4) aprender a identificar las causas y no las consecuencias para de este modo encontrar soluciones a problemas concretos; (5) generar habilidades necesarias para desarrollar el potencial personal; (6) comprender saberes y valores respecto a diversos ámbitos del desarrollo humano.

Algunos autores como Merrian y Caffarella (citados por FALASCA, 2011) y Knowles (1980) identifican las siguientes características del estudiante adulto:

- Son autónomos y autodirigidos; necesitan ser libres para autodirigirse;

- Los adultos tienen acumulados fundamentos, experiencia de vida y conocimiento relacionado con sus actividades, la familia y la educación previa ;

- Los adultos se orientan hacia lo relevante o significativo, deben ver o encontrar una razón para aprender algo;

- Los adultos se centran más en los problemas que en el aprendizaje; 
- Los adultos están motivados al aprendizaje por factores internos más que por externos.

Los objetivos de la educación permanente o de la formación a lo largo de la vida incluyen la ciudadanía activa, la realización personal y la integración social, así como aspectos relacionados con la empleabilidad. Los principios que subyacen en el aprendizaje permanente y guían su realización resaltan el papel central del estudiante, la mejora de la calidad de vida y la relevancia de las oportunidades de aprendizaje.

\section{Aprendizaje por experiencia}

El aprendizaje de la experiencia o aprendizaje experiencial es un proceso activo de construcción de conocimiento con las siguientes características:

- Articula el saber y el saber hacer. El saber (conocimientos, habilidades y actitudes) de una disciplina, profesión u oficio tiene sentido en cuanto me permite desarrollar acciones efectivas (saber hacer) para el cumplimiento de metas esperadas. El saber hacer revisado enriquecerá el cuerpo de conocimientos disciplinares, profesionales y del oficio (DÍAZ BARRIGA, 2006).

- $\quad$ Es altamente contextual. El saber sistematizado de una disciplina, profesión u oficio es la base indispensable, pero no suficiente, ya que es necesario el análisis de las distintas circunstancias en que se dan los problemas, que a su vez contribuyen a tomar decisiones de actuación respecto a otros problemas y en otras condiciones.

- $\quad$ Es socialmente apoyado. La complejidad de los problemas habituales requiere de la ayuda de otros en su resolución, quienes aportan su punto de vista sobre el problema, enriqueciendo la solución y conocimiento personal.

- Se realiza de acuerdo a objetivos, estrategias y recursos. En la resolución de problemas se ponen en juego recursos personales (conocimientos y habilidades) y sociales (otras personas), así como estrategias de intervención (combinación de acciones) de acuerdo a determinadas condiciones para llevar a cabo las soluciones de los mismos.

Gran parte del conocimiento de los seres humanos proviene de las experiencias vividas. Mientras éstas sean más numerosas, diversas y de calidad, mayores son las posibilidades de generar conocimiento. Por ejemplo, una persona que viaja, realiza alguna actividad deportiva, artística, de ayuda social o política, cuenta con un abanico de fuentes de aprendizaje más amplio que quien sólo se dedica a estudiar o trabajar.

Sin embargo, las experiencias en sí mismas no garantizan aprender de ellas. Se aprende de la experiencia en tanto: (a) se reflexione sobre la misma con la intención de 
generar acciones o soluciones a problemas y (b) se vincule a las necesidades, intereses y valores personales y colectivos, que nos constituyen como sujetos de una sociedad.

El ciclo de aprendizaje experiencias (ver esquema 1) consistente en cuatro fases:

1. Vivencia y enfrentamiento con una experiencia problemática concreta, vista desde el conocimiento previo (concepciones, creencias, cultura, formación escolar) en un contexto específico (circunstancias físicas, sociales, económicas, culturales y políticas).

2. Desarrollo de una observación reflexiva sobre el carácter de la experiencia, destacando el análisis y relación de las consecuencias de la actuación.

3. Conceptualización abstracta, donde se elaboran conceptos, ideas y teorías para la acción encaminada a la solución del problema en cuestión.

4. Experimentación activa o prueba, consistente en la aplicación de la solución pensada que se contrasta con nueva información sobre el problema, esto conduce a una nueva vivencia o problema a manera de un ciclo en espiral con el desarrollo de nuevo conocimiento.

\section{Esquema 1 - Ciclo de aprendizaje experiencial}

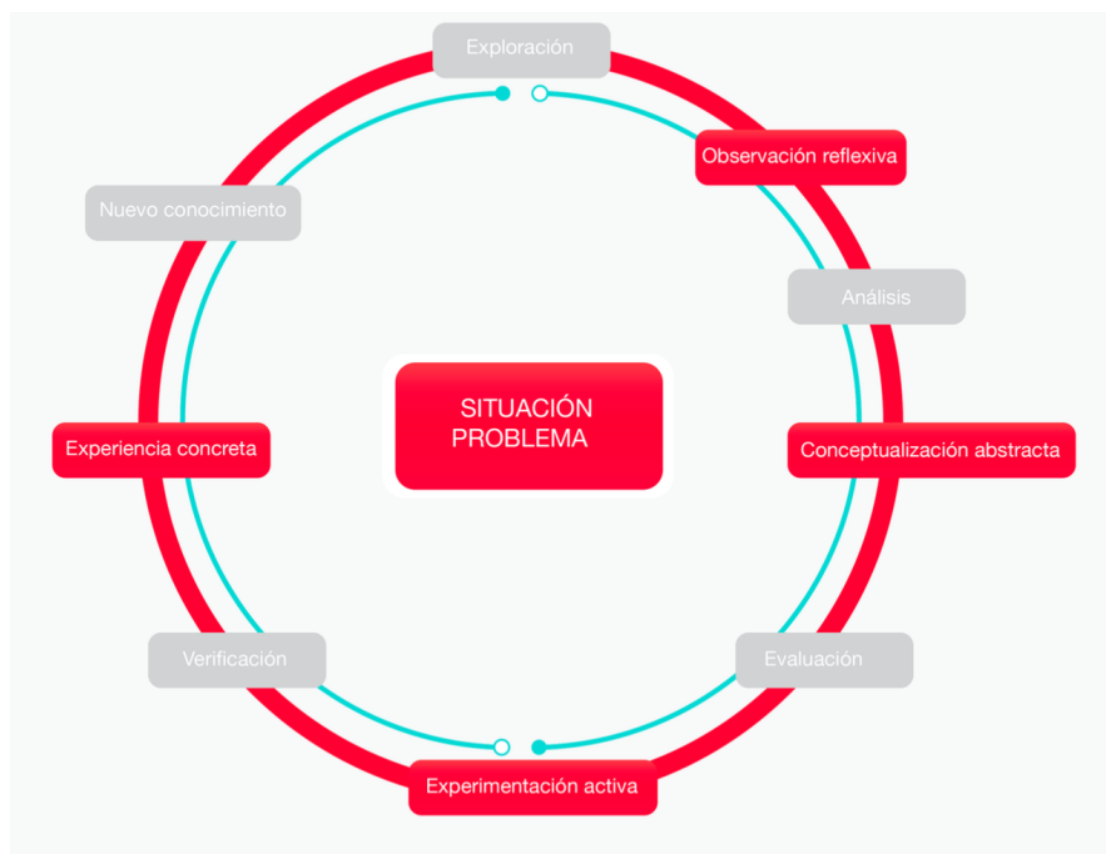

Fuente: Adaptado de Kolb (1984).

Es esencial aprender a cultivar experiencias de vida que conduzcan al desarrollo personal y a la mejora del entorno social. La educación es un proceso clave para lograrlo. Educarse va más allá de estar informado y capacitado para la realización de un trabajo. De acuerdo a la Organización de las Naciones Unidades para la Educación, la 
Ciencia y la Cultura (DELORS, 1996) la educación se sustenta en cuatro pilares: aprender a conocer, aprender a hacer, aprender a vivir juntos y con los demás y aprender a ser.

El aprendizaje de estos cuatro pilares es un proceso: (a) basado en la adaptación y transformación objetiva y subjetiva de la experiencia, (b) en torno a la resolución de conflictos por los que la persona se moviliza, (c) fuente de creación de conocimiento que (d) se gesta en la interacción social entre la persona con las necesidades de su contexto, (e) de carácter permanente al desarrollarse a lo largo de la vida y (f) holístico al abarcar todas las áreas de ésta última.

\section{Ambiente de aprendizaje en línea}

Los avances en las ciencias de la información y las nuevas propuestas pedagógicas han permitido crear entornos formativos centrados en el aprendiz. Estos entornos se caracterizan por ser sistemas interactivos, accesibles, flexibles y significativos, desde los cuales las personas pueden aprender en línea a través de internet sin requerir de la presencialidad de todos los participantes en un mismo espacio/tiempo.

Las plataformas de enseñanza son un recurso clave para cubrir dos aspectos relevantes en la educación de adultos: la cobertura y la calidad. Respecto del primero, este recurso contribuye a llegar a zonas distantes, minimiza los costos al evitar el desplazamiento de los participantes y con ello, contribuye a dar viabilidad a los proyectos. En cuanto al segundo, al normar con criterios generales precisos pero flexibles, garantiza que todos los estudiantes tengan un acceso equitativo y controlado respecto de la calidad de los materiales a través de los cuales se pretende formar.

La plataforma de enseñanza es el articulador del ambiente virtual de aprendizaje heurístico, entendido como un entorno creado ex profeso que privilegia las teorías y los principios del aprendizaje significativo, desarrollando las teorías construccionistas, centrando los programas en los participantes, favoreciendo su capacidad intuitiva e inventiva Ruiz-Velasco (2004-2005). En la plataforma se integran las herramientas y recursos necesarios para gestionar, administrar, organizar, coordinar, diseñar e impartir cursos a través de Internet.

Los elementos que componen el ambiente virtual de aprendizaje son:

- el sistema de administración de contenidos y usuarios

- el diseño instruccional que se ha concebido para poner en común los contenidos

- el formato de la tutoría para guiar a los estudiantes en el camino del aprendizaje 
Por lo que se refiere al tiempo en el ambiente virtual, se requiere un manejo acertado, sobre todo el dedicado a la interacción, ya que es el que en mayor medida contribuye a la conformación de comunidades de aprendizaje, entendidas como un conjunto de relaciones sociales, unidas por un interés común o circunstancias compartidas, que se dan en el ciberespacio y que representan una extensión de la vida cotidiana (TESOURO; PUIGGALÍ, 2006; CABRERO et al. 2008).

Los ambientes virtuales proveen a los usuarios de estímulos a través de medios múltiples, así por ejemplo recursos como el video, el audio y el texto, apelan a los sentidos del usuario de tal modo que lo llevan a tener una gama de percepciones de la realidad, al tiempo que lo motivan para explorar y acercarse a los diversos recursos del ambiente.

Para definir el diseño instruccional se retoma a Gustafson y Branch (2007) quienes lo conceptualizan como el proceso sistemático empleado para desarrollar programas educativos y de capacitación de forma consistente y confiable. El modelo de diseño curricular se propone en un ciclo de cuatro etapas, que inicia con activar el conocimiento previo relevante. Se facilita el aprendizaje cuando la instrucción lleva a los estudiantes a recordar, relacionar, describir, o aplicar los conocimientos de la experiencia previa y sirve como base para el nuevo conocimiento. En un segundo momento, se proponen actividades para la práctica o demostración del nuevo conocimiento adquirido. En una tercera etapa del ciclo, el aprendizaje se facilita cuando se brinda la oportunidad a los alumnos para aplicar los nuevos conocimientos a situaciones específicas. Finalmente, un ciclo de aprendizaje se completa cuando los alumnos tienen la oportunidad de integrar los nuevos conocimientos y destreza en sus actividades cotidianas (REISER; DEMPSEY, 2007).

\section{Propuesta}

\section{Configuración del programa}

La asignatura Estrategias de aprendizaje y habilidades digitales se incorporó a la estructura curricular de los 14 programas de licenciatura enfocados a adultos trabajadores en la Universidad del Valle de México. A partir del rediseño de dichas licenciaturas realizado en el año 2014, éstas se componen por 41 asignaturas distribuidas en 9 ciclos de 14 semanas y agrupadas en tres áreas: básica, profesional y desarrollo ejecutivo. La modalidad de estos programas es mixta, en ella los estudiantes 
cursan menos de 2400 horas acompañados por un docente en asignaturas de diverso formato como a continuación se enlistan:

- Asignaturas presenciales: las horas con docente se imparten en forma presencial en instalaciones físicas del campus

- Asignaturas mixtas o blended learning: el 50\% de las horas con docente se imparten de forma presencial en las instalaciones físicas de la universidad y el $50 \%$ se imparten a distancia mediante la plataforma de enseñanza en línea

- Asignaturas en línea: el total de las horas con docente se imparten a distancia mediante la plataforma.

Los tres tipos de asignatura se distribuyen de forma equilibrada en el curriculum de tal suerte que:

a) Los ciclos de primero a cuarto se compone de dos asignaturas presenciales y dos blended o mixtas

b) Los ciclos de quinto a noveno se componen de dos asignaturas presenciales, dos asignaturas blended y una asignatura en línea

La asignatura objeto de este documento, está organizada en 7 semanas que se conforman por una sesión en línea y una presencial, durante los cuales se abordan cuatro temas conforme a la siguiente tabla:

Tabla 1 - Estructura del curso

\begin{tabular}{|lll|}
\hline Unidad & Contenido & Duración \\
\hline Unidad 1 & Habilidades de aprendizaje en línea & 2 semanas \\
\hline Unidad 2 & Características del aprendizaje adulto & 2 semanas \\
\hline Unidad 3 & Estrategias de aprendizaje adulto & 2 semanas \\
\hline Unidad 4 & Evaluación y portafolios de evidencias & 1 semana \\
\hline
\end{tabular}

Fuente: Elaboración propia.

Cada una de las cuatro unidades del curso se integra por los subtemas que se ilustran a continuación en el Esquema 2. 


\section{Esquema 2 - Mapa de contenido de la asignatura}

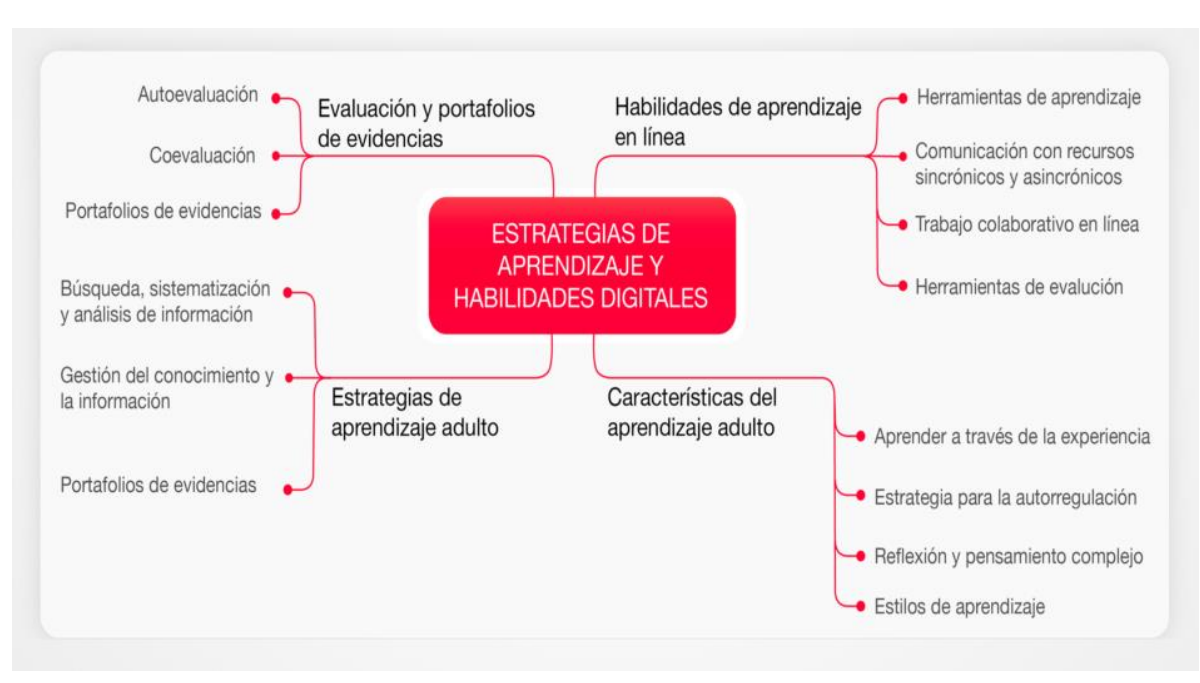

Fuente: Elaboración propia.

En las primeras dos semanas se abordan habilidades para el aprendizaje en línea, mismas que incluyen la exploración de herramientas de aprendizaje, el uso de recursos de comunicación sincrónica y asincrónica mediados por tecnología, dinámicas de trabajo colaborativo en línea y herramientas para la auto, hetero y co evaluación.

Las semanas tres y cuatro se profundiza en las características del aprendizaje adulto y las experiencias que permiten lograr un aprendizaje significativo en esta etapa de la vida, entre ellas encontramos el aprender mediante la experiencia de vida, las estrategias para la autorregulación, las habilidades de reflexión y pensamiento complejo y la identificación de los diversos estilos de aprendizaje. Esta unidad pretende otorgar al estudiante recursos de trabajo intelectual para optimizar su desempeño, habilidades $\mathrm{y}$ recursos propios para facilitar su permanencia exitosa en los estudios de licenciatura.

Las semanas cinco y seis, se exploran estrategias clave para sistematizar el estudio en la vida adulta, incorporando recursos tecnológicos para la organización de información y gestión de conocimiento, al tiempo que se trabaja en las habilidades de comunicación oral y escrita para la transmisión efectiva de mensajes en los ambientes de estudio y laboral.

Finalmente en la semana siete el estudiante se familiariza con la evaluación como parte del proceso de aprender y como elemento que apoya el compromiso con el propio aprendizaje. Así, reconocer el valor de la auto y coevaluación o evaluación entre pares, contribuye al desarrollo de habilidades de meta aprendizaje y compromete al estudiante con su propio desarrollo. La conformación de un portafolios de evidencias 
pone de manifiesto los logros durante el curso y promueve la reflexión del estudiante sobre su aprendizaje.

Por lo que se refiere a la evaluación de las asignaturas en las licenciaturas dirigidas a adultos, es de carácter formativo, por lo cual las actividades realizadas en el aula presencial, en la plataforma de enseñanza y de manera independiente forman parte de la evaluación del desempeño y logro del estudiante. Igualmente se propicia la autoevaluación y la evaluación entre pares con el propósito de desarrollar habilidades críticas, reflexivas y de metaconocimiento.

A manera de integración, se solicita la elaboración de un trabajo final que permite contar con las evidencias del aprendizaje logrado por el estudiante, para tal efecto se desarrolla un portafolios que incluye los documentos elaborados en el curso, un escrito final en el cual refleje las competencias para la reflexión y el autoanálisis, así como las rúbricas comentadas de tales tareas.

\section{Metodología de trabajo}

La asignatura se imparte en la modalidad blended learning, es decir se tienen sesiones semanales en el campus y en la plataforma de aprendizaje. Las sesiones tienen una duración de tres horas en ambos casos. Las estrategias usadas son principalmente problemas y casos en los cuales se presentan diversos aspectos o temas de la asignatura.

El trabajo presencial implica sobretodo la participación activa del estudiante en una dinámica que pretende que construyan ellos mismos el conocimiento a partir de la revisión de materiales, resolución de dudas y trabajo colaborativo.

Las sesiones en línea cuentan con actividades estructuradas que permiten abordar el contenido de forma autónoma y con apoyos puntuales por parte del docente. Las actividades semanales en línea tienen un componente individual y uno colaborativo.

En la plataforma de aprendizaje el estudiante encuentra los siguientes elementos:

- Presentación y mapas de contenido

- Competencia a desarrollar

- Situación problema por unidad o semana

- Unidades de contenido

- Actividades de demostración y aplicación

- Actividades de integración y reflexión individuales y colaborativas

- Fuentes de información en línea obligatorias y complementarias

- Recursos formativos de profundización 
- Glosarios

- Estrategia de evaluación

- Cuestionario de valoración de la asignatura

\section{Ambiente de aprendizaje}

Se generó un ambiente en el que el participante encontrara y construyera recursos para dar sentido a sus ideas que le permitiera desarrollar soluciones significativas a los problemas y retos del contenido para lo cual se propuso:

- abordar el aprendizaje a través de problemas, casos, proyectos, entre otros;

- considerar en las estrategias y diseño las características de los usuarios en lo individual y lo colectivo;

- propiciar el aprendizaje colaborativo, la comunicación y el trabajo en grupo;

- diversificar los recursos de aprendizaje audiovisuales, interactivos y autogenerativos.

El entorno de aprendizaje se publicó en una plataforma o Learning Content Management System (LCMS) Blackboard, misma que permite el acceso a contenidos, gestión de recursos y usuarios, provee de herramientas de comunicación sincrónica y asincrónica. Además de generar contenidos e impartirlos, es posible almacenarlos, distribuirlos y reutilizarlos. Blackboard cuenta con herramientas para que la comunidad virtual interactúe y genere no solamente los aprendizajes requeridos, sino que como grupo sea capaz de trascender los límites curriculares y generar sus propios conocimientos a través de diversos espacios entre los cuales se encuentran los siguientes:

- Espacio de documentación, donde se ubican los contenidos en diversos formatos

- Espacio de comunicación e intercambio, cuenta con herramientas de comunicación sincrónica y asincrónica, como son el chat, el correo, los foros y el tablón de novedades, siendo estos dos últimos los que se usan mayormente.

- Espacio de trabajo, constituido por aquellos elementos que propician el trabajo en línea como las evaluaciones y ejercicios en tiempo real y los foros que facilitan el trabajo colaborativo

- Espacio de evaluación, en el que el participante deposita o realiza los trabajos para evaluación y el profesor o tutor los comenta y califica

En lo referente al diseño instruccional uno de los elementos más importantes del modelo que se propone es el trabajo basado en problemas y casos donde a partir del planteamiento de situaciones reales, el estudiante elabora un diagnóstico, se muestran los componentes de la situación y se hace uso de recursos para resolver el problema o 
desarrollar la actividad. Se integran actividades individuales y colaborativas, generando escenarios para la resolución individual y colectiva de situaciones complejas en las que se recuperan experiencias laborales y de vida de los estudiantes. La evaluación es formativa en tanto se deben ir resolviendo etapa por etapa los problemas planteados, además de que la auto y la coevaluación juegan un papel relevante en la evaluación en su conjunto

En cuanto a la estructura de la asignatura el modelo incluye los siguientes elementos:

- Presentación y mapas de contenido

- Competencia a desarrollar

- Estrategias de enseñanza

- Situación problema general y/o por unidad

- Unidades de contenido

- Actividades obligatorias a desarrollar

- Actividades de reforzamiento

- Estrategia de evaluación (auto - cohetero)

- Fuentes de información en línea obligatorias y complementarias

- Recursos formativos de profundización

- Glosarios

- Cuestionarios de evaluación del aprendizaje por unidad de contenido

- Cuestionario de valoración de la asignatura

En lo referente a los materiales propuestos y desarrollados para el eficiente funcionamiento de este modelo se incluye lo siguiente:

- Situaciones, problemas y casos viñeta con preguntas de conocimiento, aplicación y resolución: en formato flash y descargable para smartphone, video, podcast, comic, ilustración

- Unidades de contenido: objetos de aprendizaje bajo el estándar scorm, libros electrónicos descargables en pdf, para visualizarlos en tableta electrónica o en la pc.

- Actividades individuales y/o colectivas de socialización y resolución colaborativa: paquetes scorm; web quest, wikis, blogs, sitios colaborativos, repositorios de podcast y video, sitios colaborativos y redes sociales

- Actividades de hetero, auto y co evaluación: e-rúbrica, cuestionarios, cruces de columna, combos, líneas de tiempo, mapas mentales, mapas conceptuales 


\section{Conclusiones}

La recuperación de estrategias de aprendizaje y el desarrollo de habilidades digitales son requisitos para un reingreso exitoso a la universidad, sobre todo en un modelo flexible donde se pretende que el estudiante adulto asuma plenamente la responsabilidad de un aprendizaje autónomo basado en la experiencia laboral y de vida.

La propuesta se basa en incorporar la citada experiencia a lo largo del itinerario formativo mediante casos, problemas y reflexiones que la vinculan al nuevo conocimiento para transferir así lo aprendido a situaciones propias de su entorno laboral presente o futuro.

Al generar una multiplicidad de formatos y vías de acceso a los materiales didácticos se espera que los estudiantes tengan las condiciones necesarias para realizar las actividades en el tiempo adecuado para ellos, asimismo se diseñaron ejercicios que pretenden acercarlo de forma lúdica a conceptos y procesos definidos que eventualmente incorporará a su propia práctica de aprendizaje.

La asignatura Estrategias de aprendizaje y habilidades digitales es la primera de un total de 41 materias, de ahí la importancia de su diseño y planificación en el resultado en primera instancia de la adecuada reinserción escolar de los estudiantes adultos y en segunda, de la modelación de su modo de aprender y dominio de las herramientas digitales propias de la universidad y aplicadas a su profesión.

ABSTRACT: This document describes a didactic sequence to develop learning skills and the way to use the technology in adult students returning to college. The proposal integrates online digital resources like e-books, stories, memory games and exercises, while the presencial classes integrate collaborative learning and active participation to ensure the integration of this kind of student into the college environment. The sequence will start this school year with at least 2,000 students of new enrollment, are expected that, with the help of this subject, students achieve more efficiently inserted to college after a while of begin their educational process.

KEYWORDS: Blended learning. Situated learning. Higher education adult learning.

\section{REFERENCIAS}

ASOCIACIÓN NACIONAL DE UNIVERSIDADES E INSTITUCIONES DE EDUCACIÓN SUPERIOR [ANUIES]. Anuario Digital Educación Superior Licenciatura: 2012-2013. 2015. Disponible en: <http://www.anuies.mx/iinformaciony-servicios/informacion-estadistica-de-educacion-superior/anuario-estadistico-deeducacion-superior>. Acceso en: 07 jul. 2014. 
CABRERO, B. et al. Análisis de los patrones de interacción y construcción del conocimiento en ambientes de aprendizaje en línea: una estrategia metodológica.

Revista Electrónica de Investigación Educativa, Ensenada, v.10, n.1, p.1-19, 2008. Disponible en: <http://redie.uabc.mx/index.php/redie/article/view/179>. Acceso en: 12 sept. 2014.

DELORS, J. et al. Los cuatro pilares de la educación. En: UNESCO. La educación encierra un tesoro: informe a la UNESCO de la Comisión internacional sobre la educación para el siglo XXI. Madrid: Santillana: UNESCO, 1996. p.91-103. Disponible en:

<http://redeca.uach.mx/contexto/Los\%204\%20pilares\%20de\%20la\%20educacion.pdf $>$. Acceso en: 12 sept. 2014.

DÍAZ BARRIGA, A. F.; HERNÁNDEZ, R. G. Estrategias docentes para un aprendizaje significativo: una interpretación constructivista. México: McGraw, 2006.

FALASCA, M. Barriers to adult learning: bridging the gap. Australian Journal Of Adult Learning, Underdale, v.51, n.3, p.583-590, 2011.

GUSTAFSON, K. L.; BRANCH, R. M. What is instructional design? En: REISER, R. A.; DEMPSEY, J. V. (Comp.). Trends and issues in instructional design and technology. New Jersey: Pearson, 2007. p.10-16.

INSTITUTO NACIONAL DE ESTADÍSTICA Y GEOGRAFÍA [INEGI]. Anuario de estadísticas por entidad federativa 2012. México, 2013. Disponible en:

<http://www.inegi.org.mx/prod_serv/contenidos/espanol/bvinegi/productos/integracion/ pais/aepef/2012/Aepef2012.pdf>. Acceso en: 07 jul. 2014.

KNOWLES, M. S. The modern practice of adult education: andragogy versus pedagogy. Cambridge: Prentice Hall, 1980.

KOLB, D. A. The process of experiential learning: experiential learning: experience as the source of learning and development. New Jersev: Prentice-Hall, 1984. p.20-38. Disponible en: <http://academic.regis.edu/ed205/kolb.pdf>. Acceso en: 18 abr. 2014.

REISER, R. A.; DEMPSEY, J. V. Trends and issues in instructional design and technology. New Jersey: Pearson, 2007.

RUÍZ-VELASCO, E. Ambiente de aprendizaje heurístico. Tecnología y Comunicación Educativas, México, v.18, n.40, p.68-93, jul. 2004-jun. 2005. Disponible en:

$<$ http://investigacion.ilce.edu.mx/stx.asp?id=1631\&db=\&ver=>. Acceso en: 22 abr. 2014.

TESOURO, C. M.; PUIGGALÍ, A. J. Las comunidades virtuales y de conocimiento en el ámbito educativo. Píxel.Bit: Revista de medios y educación, Sevilla, v.28, 2006.

Disponible en:

<http://www.sav.us.es/pixelbit/pixelbit/articulos/n28/n28art/art2810.htm>. Acceso en: 03 feb. 2014.

UNIVERSIDAD DEL VALLE DE MÉXICO. Resultados encuestas, rediseño de carreras. México, 2011. 